\title{
Stability analysis and controller synthesis for hybrid dynamical systems*
}

\author{
W.P.M.H. Heemels, B. De Schutter, J. Lunze, and M. Lazar
}

If you want to cite this report, please use the following reference instead:

W.P.M.H. Heemels, B. De Schutter, J. Lunze, and M. Lazar, "Stability analysis and controller synthesis for hybrid dynamical systems," Philosophical Transactions of the Royal Society A, vol. 368, no. 1930, p. 4937-4960, Nov. 2010.

Delft Center for Systems and Control

Delft University of Technology

Mekelweg 2, 2628 CD Delft

The Netherlands

phone: +31-15-278.24.73 (secretary)

URL: https: / / www.dcsc.tudelft.nl 


\title{
Stability analysis and controller synthesis for hybrid dynamical systems $\dagger$
}

\author{
By W.P.M.H. Heemels, B. De Schutter, J. Lunze, and M. Lazar $\ddagger$
}

Wherever continuous and discrete dynamics interact, hybrid systems arise. This is especially profound in many technological systems in which logic decision making and embedded control actions are combined with continuous physical processes. Also for many mechanical, biological, electrical, and economical systems the usage of hybrid models is indispensable to adequately describe their behavior. To capture the evolution of these systems, mathematical models are needed that combine in one way or another the dynamics of the continuous parts of the system with the dynamics of the logic and discrete parts. These mathematical models come in all kinds of variations, but basically consist of some form of differential or difference equations on the one hand and automata or other discrete-event models on the other hand. The collection of analysis and synthesis techniques based on these models forms the research area of hybrid systems theory, which plays an important role in the multi-disciplinary design of many technological systems that surround us. This paper presents an overview from the perspective of the control community on modeling, analysis, and control design for hybrid dynamical systems and surveys the major research lines in this appealing and lively research area.

Keywords: Hybrid systems, piecewise affine systems, stability, control design

\section{Introduction}

Basically, hybrid systems theory studies the behavior of dynamical systems that are modeled through mathematical formalisms that involve both continuous models such as differential or difference equations, and discrete models such as finite state machines or automata. The term "hybrid systems" expresses that the dynamical systems at hand describe a mixture of heterogeneous modeling formalisms, namely continuous models as adopted in mathematical control theory and discrete models as used in computer science. In this particular context, the term was used for the first time in (Witsenhausen, 1966).

A textbook example of a hybrid system considers the regulation of the temperature in a house. In a simplified description, the heating system is assumed either to work at its maximum power or to be turned off. This system can operate in two modes: "on" and "off". In each mode of operation (given by the discrete state $q \in\{\mathrm{on}$, off $\}$ ) the evolution of the temperature $T$ can be described by a different

$\dagger$ The authors are grateful for the permission of Cambridge University Press to use parts of their chapters in (Lunze and Lamnabhi-Lagarrigue, 2009) in this overview paper.

$\ddagger$ Maurice Heemels and Mircea Lazar are with Eindhoven University of Technology, The Netherlands, \{m.heemels,m.lazar\}@tue.nl. Bart De Schutter is with Delft University of Technology, The Netherlands, b.deschutter@tudelft.nl. Jan Lunze is with Ruhr-Universität Bochum, Germany, lunze@atp.rub.de. 


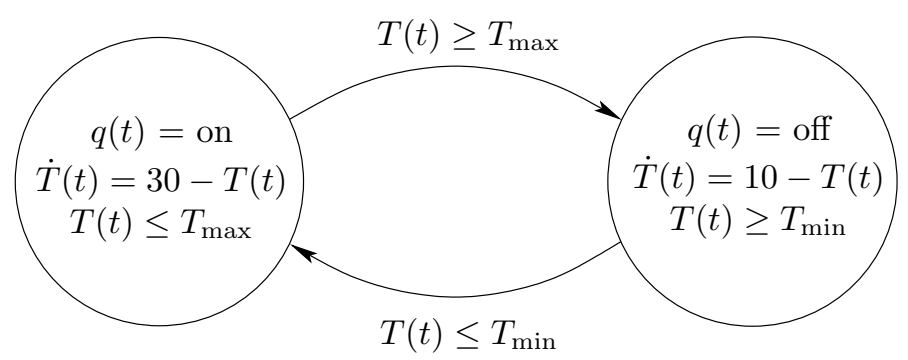

Figure 1. Model of a temperature control system.

differential equation. This is illustrated in Fig. 1 in which each mode corresponds to a node of a directed graph, while the edges indicate the possible discrete state transitions. As such, this system has a hybrid state $(q, T)$ consisting of a discrete state $q$ taking the discrete values "on" and "off" and a continuous state $T$ taking values in the real numbers.

Clearly, the value of the discrete state $q$ affects the evolution of the continuous state $T$ as a different vector field is active in each mode. Vice versa, the switching between the two modes of operation is controlled by a logical device (the embedded controller) called the thermostat and depends on the value of the continuous state $T$. The mode is changed from "on" to "off" whenever the temperature $T$ reaches the value $T_{\max }=21$ (determined by the desired temperature, which is, say, 20 degrees Celcius). Vice versa, when the temperature $T$ reaches a minimum value $T_{\min }=19$, the heating is switched "on."

This example already shows some of the main features of hybrid systems:

- The system has a hybrid state consisting of a discrete state $q$ (taking a finite number of values only) and a continuous state $T$.

- The continuous behavior of the system depends on the discrete state, i.e., depending whether the mode is "on" or "off" a different dynamics $\dot{T}(t)=$ $30-T(t)$ or $\dot{T}(t)=10-T(t)$, respectively, governs the evolution of the temperature $T$.

- The changes of the discrete state $q$ are determined by the continuous state $T$ and different conditions on $T$ might trigger the change of the discrete state (e.g., when the discrete state is "on," $T=T_{\max }=21$ triggers the mode change, while $T=T_{\min }=19$ triggers the change when the discrete state is "off.")

Although the above example is extremely simple, it reflects already the interactions between embedded controllers with a discrete character on the one hand and physical processes with a continuous character on the other as are omnipresent in technological systems such as wafer scanners, electron microscopes, copiers, robotic systems, automobiles, and many more. The growing complexity of technological systems urged for a deeper understanding of the complicated interaction between the discrete and continuous dynamics in the early nineties. As a result, the academic world started investigating the rich behavior that is produced by these hybrid systems in the mid 90s. This is evidenced by the start of a workshop series (Henzinger and Sastry, 1998) and many special issues being published in that period (Antsaklis and Nerode, 1998; Morse et al., 1999; Pnueli and Sifakis, 1995). 
In technological (man-made) systems the digital and logic (embedded control) aspects are typically brought in by design in order to control the physics and mechanics of the system. However, hybrid modeling and systems theory is not only useful within the technological domain. Many physical processes exhibiting both fast and slow changing behaviors can often be well described by using (simple) hybrid models. For instance, in non-smooth mechanics (Brogliato, 1996), the evolution of impacting rigid bodies can be captured by hybrid models. Indeed, as the impacts occur at a much smaller time scale than the unconstrained motion, the behavior can be described well by introducing discrete events (corresponding to impacts) and discrete (reset) actions in a smooth model. Also the vector fields defining the behavior of the system might be different over time as they depend crucially on the fact whether a contact is active or not. The dynamics of a robot arm moving freely in space is completely different from the situation in which it is striking the surface of an object. Other hybrid examples in mechanics include motion systems with friction models that distinguish between stick and slip modes, backlash in gears, dead zones in cog wheels, etc. Clearly, examples are not only found in the mechanical domain. Nowadays switches such as thyristors and diodes are used in electrical networks for a wide variety of applications in both power engineering and signal processing. Examples include switched-capacitor filters, modulators, analog-to-digital converters, power converters, and choppers. As an example, consider diodes, which in the ideal case behave as elements with two (discrete) modes: the blocking mode and the conducting mode. Mode transitions for diodes are governed by state events, where currents or voltages change their sign. This indicates that hybrid modeling and analysis techniques offer an attractive perspective on these switched circuits as well. Also many biological and chemical systems can often be efficiently described by hybrid models, as is clear from various papers in this special issue.

Given the wealth of applications and the urgent need for more systematic design methods for technological systems, the enormous interest in the field of hybrid systems does not come as a surprise. Various interesting results are obtained over the years, although many problems remain to be unsolved at present. In this paper we intend to provide an introduction to hybrid dynamical systems and review the main research lines from the perspective of the control community. For more details on these research lines we will present various references for further reading. In this manner this paper can be used as a starting point for research in the hybrid systems area.

\section{A selection of hybrid modeling formats}

The thermostat example has already shown some of the basic ingredients that are needed to properly model hybrid systems. A proper modeling format must involve (at least) the description of the evolution of both continuous-valued signals (temperatures, positions, velocities, currents, voltages, etc.) and discrete-valued signals (operation mode, position of a switch, alarm on or off, etc.) over time and their mutual influence. Below we will discuss various hybrid models that indeed include these characteristics.

Preprint for Royal Society 


\section{(a) Hybrid automata}

A widely used model for hybrid systems, is the hybrid automaton model that combines finite state machines / automata as adopted in computer science, and differential equations as used in mathematical control theory. This model was formalized for the first time by (Alur et al., 1995) and in the meantime many variations and ramifications have been proposed in the literature, see, e.g., (Alur and Henzinger, 1997; Cassandras and Lafortune, 1999; Lynch et al., 1996; Lygeros et al., 1998; Branicky et al., 1998). There are also stochastic versions of hybrid automata, see, e.g., (Cassandras and Lygeros, 2007) and the references therein. Here we present a basic deterministic version without external inputs for brevity.

Definition A hybrid automaton $H$ is an 8-tuple $H=(Q, X, f, \operatorname{Init}, \operatorname{Inv}, E, G, R)$ with

- $Q=\left\{q_{1}, \ldots, q_{N}\right\}$ is a finite set of discrete states;

- $X=\mathbb{R}^{n}$ is a set of continuous states;

- $f: Q \times X \rightarrow X$ is a vector field;

- Init $\subseteq Q \times X$ is a set of initial states;

- Inv : $Q \rightarrow P(X)$ describes the invariants;

- $E \subseteq Q \times Q$ is a set of edges;

- $G: E \rightarrow P(X)$ is a guard condition;

- $R: E \rightarrow P(X \times X)$ is a reset map.

Note that $P(X)$ is the power set of $X$, i.e., the collection of all subsets of $X$. Hence, the guard condition $G$ specifies for each mode transition or edge a subset of $X$. The (hybrid) state variable of the system $H$ is given by $(q, x) \in Q \times X$.

Just to indicate briefly how this dynamical system behaves, we give a short, rather informal description. The initial hybrid state $\left(q_{0}, x_{0}\right)$ of "trajectories" of a hybrid automaton must be contained in the initial set Init. From this hybrid state the continuous state $x$ evolves according to the differential equation

$$
\dot{x}=f\left(q_{0}, x\right) \quad \text { with } x(0)=x_{0}
$$

while the discrete state $q$ remains constant: $q(t)=q_{0}$. The continuous evolution can go on as long as $x$ stays in $\operatorname{Inv}\left(q_{0}\right)$. If at some point the continuous state $x$ reaches the guard $G\left(q_{0}, q_{1}\right)$, we say that the transition is enabled. The discrete state may then change to $q_{1}$, and the continuous state jumps from the current value $x^{-}$to a new value $x^{+}$that satisfies $\left(x^{-}, x^{+}\right) \in R\left(q_{0}, q_{1}\right) \dagger$. After this transition, the continuous evolution resumes and the whole process is repeated.

$\dagger$ Sometimes the resets are given as $x^{+}=r_{\left(q_{0}, q_{1}\right)}\left(x^{-}\right)$, which can be incorporated in the reset map by taking $R\left(q_{0}, q_{1}\right)=\left\{\left(x, r_{\left(q_{0}, q_{1}\right)}(x)\right) \mid x \in \underset{X}{X}\right\}$. 


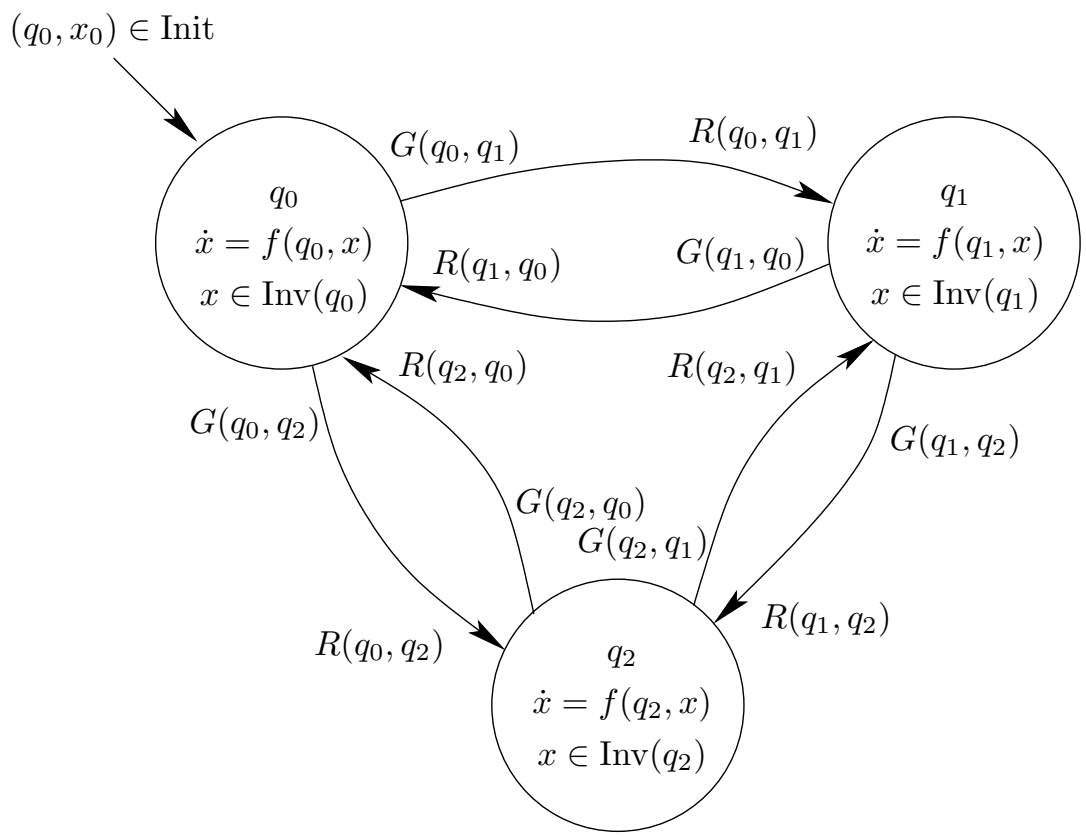

Figure 2. Schematic representation of a hybrid automaton with 3 discrete states. Each node of the directed graph represents a mode (operating point) given by a set of differential equations. The arrows indicate the possible discrete transitions that correspond to a change of the mode, which can be accompanied by resets of the continuous state variable.

\section{(b) Switched systems}

A quite general class of hybrid systems, although less general than hybrid automata, concerns switched systems given by

$$
\dot{x}(t)=f_{q(t)}(x(t))
$$

where $x \in \mathbb{R}^{n}$ denotes the state and $q: \mathbb{R}_{+} \rightarrow\{1, \ldots, N\}$ is the switching signal that determines which vector field $f_{q}$ with $q \in\{1, \ldots, N\}$ is active at time $t \in \mathbb{R}_{+}$. For a fixed $q,(2.1)$ describes a non-switched system, which is sometimes called a subsystem of the switched system. In case $f_{q}(x)=A_{q} x$ with $A_{q} \in \mathbb{R}^{n \times n}$, we speak about a switched linear system. The switching can depend on time only, but it can also be a function of the state $x(t)$ at time $t$ or of an external input, and it can even have memory in it. In particular, when the switching only depends on the state variable $x(t)$ at the present time $t$, one speaks of discontinuous dynamical systems or piecewise smooth systems.

(c) Discontinuous dynamical systems and piecewise affine systems

A typical example of a discontinuous dynamical system is the system below, which switches between two (smooth) vector fields as a result of inequalities in the state variable:

$$
\dot{x}(t)=f(x(t))= \begin{cases}f_{-}(x(t)), & \text { if } \phi(x(t)) \leq 0 \\ f_{+}(x(t)), & \text { if } \phi(x(t)) \geq 0\end{cases}
$$


For this system the state space is separated into two parts by a hyper-surface defined by $\phi(x)=0$. On one side of the surface $\mathcal{C}_{+}:=\left\{x \in \mathbb{R}^{n} \mid \phi(x)>0\right\}$ the dynamics $\dot{x}=f_{+}(x)$ holds, on the opposite side $\mathcal{C}_{-}:=\left\{x \in \mathbb{R}^{n} \mid \phi(x)<0\right\}$ the dynamics $\dot{x}=f_{-}(x)$ is valid. Hence, one can also consider this system as a differential equation with a discontinuous right-hand side (Filippov, 1988). A distinctive difference with hybrid automata is that the state variable $x(t)$ does not have any discontinuities.

If the discontinuous dynamical system has affine dynamics in each region and the regions are polytopic (i.e., given by a finite number of linear or affine inequalities), we obtain the well-studied class of piecewise affine (PWA) systems (Sontag, 1981, 1996), which can be considered both in a discrete or continuous-time formulation. Continuous-time PWA systems are described by

$$
\dot{x}(t)=A_{q} x(t)+B_{q} u(t)+f_{q} \quad \text { when }\left[\begin{array}{l}
x(t) \\
u(t)
\end{array}\right] \in \mathcal{C}_{q}
$$

for the operation modes $q \in\{1, \ldots, N\}$, where $\mathcal{C}_{1}, \ldots, \mathcal{C}_{N}$ are convex polyhedra in the input/state space $\mathbb{R}^{n+m}$ with non-overlapping interiors. The variables $u(t) \in$ $\mathbb{R}^{m}$ and $x(t) \in \mathbb{R}^{n}$ denote the input and state respectively, at time $t \in \mathbb{R}_{+} \cdot A_{q} \in$ $\mathbb{R}^{n \times n}, B_{q} \in \mathbb{R}^{n \times m}$ and $f_{q} \in \mathbb{R}^{n}$ are constant matrices for $q \in\{1, \ldots, N\}$.

PWA systems have been studied extensively as they form the "simplest" extension of linear systems that can still approximate nonlinear (smooth and some non-smooth) models with arbitrary accuracy and are capable of handling some hybrid phenomena, as is shown in the discrete-time setting in (Heemels et al., 2001).

For other classes of hybrid models such as mixed logical dynamical (MLD) models (Bemporad and Morari, 1999), complementarity systems (van der Schaft and Schumacher, 1996, 1998), hybrid inclusions or impulsive systems (Goebel et al., 2004; Goebel and Teel, 2006; Haddad et al., 2006), timed automata (special case of hybrid automata) (Alur and Dill, 1994), and many others, see e.g. (Lunze and Lamnabhi-Lagarrigue, 2009; van der Schaft and Schumacher, 2000).

\section{Stability analysis}

The analysis of the stability property for hybrid systems received ample attention over the years, see, e.g., the book (Liberzon, 2003) or one of the surveys (Lin and Antsaklis, 2009; Shorten et al., 2007; Liberzon and Morse, 1999; DeCarlo et al., 2000). We will focus here mostly on the stability and stabilization of switched and discontinuous dynamical systems, but we will also indicate some results for other classes of hybrid models towards the end of this section. Loosely speaking, global asymptotic stability (GAS) (of the origin 0) of switched systems as in (2.1) or discontinuous dynamical systems as in (2.2) means that for each initial state $x(0)$ the corresponding solution trajectories $x(t)$ satisfy $\lim _{t \rightarrow \infty} x(t)=0$ (so-called attractivity) and that solutions starting close to the origin 0 (i.e., $x(0)$ is close to 0) remain close to 0 (so-called Lyapunov stability). See the textbook (Khalil, 1996) for more details on stability concepts.

Before we start surveying stability results for switched systems one should first formally define what is meant by the solution trajectories of the models under study, as is also reflected by the (loose) definition of GAS just given as it requires certain properties of the solutions of the system. In addition, some well-posedness 

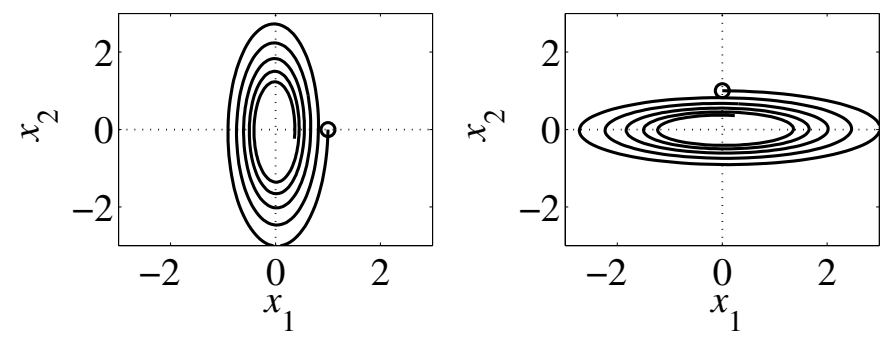

Figure 3. Behavior of the stable linear submodels $\dot{x}_{1}=A_{1} x$ (left) and $\dot{x}_{2}=A_{2} x$ (right), where the initial state is denoted by the circle $\circ$.

properties in the sense of existence (and maybe also uniqueness) of solutions given an initial condition might be convenient to make the stability property meaningful. Clearly, the adopted solution concept will have an influence on the stability notion. In particular, one has to be careful with certain hybrid phenomena such as Zenoness and sliding modes that do not appear in smooth systems. Zenoness indicates that there are an infinite number of discrete events (switching of discrete mode or resets of state variables) in a finite length time interval, while sliding modes occur in discontinuous dynamical systems such as (2.2) when the vector fields on both sides of the switching surface point towards each other (Filippov, 1988). In the latter case, infinitely fast chattering between both dynamics might occur resulting in a motion ("sliding") along the switching surface. Clearly, if Zenoness and/or sliding modes occur one has to be careful with the analysis, see, e.g., (Liberzon, 2003) and (Heemels and Weiland, 2008) in which this is demonstrated for the stability problem in case sliding modes are present. For overviews on solution concepts and well-posedness the reader might consult (Heemels et al., 2003; Cortes, 2008; Lunze and Lamnabhi-Lagarrigue, 2009).

Interestingly, stability of hybrid or switched systems cannot be tackled by just investigating stability of the subsystems only. This is illustrated by the following example taken from (Branicky et al., 1998). Consider

$$
\dot{x}=\left\{\begin{array}{ll}
A_{1} x & \text { if } x_{1} x_{2} \leq 0 \\
A_{2} x & \text { if } x_{1} x_{2}>0
\end{array} \text { with } A_{1}=\left(\begin{array}{cc}
-1 & 10 \\
-100 & -1
\end{array}\right) \text { and } A_{2}=\left(\begin{array}{cc}
-1 & 100 \\
-10 & -1
\end{array}\right)\right.
$$

By inspection of the eigenvalues of $A_{1}$ and $A_{2}$, which for both matrices are $\lambda_{1,2} \approx$ $-1 \pm 31.62 i$, one can see that both of the dynamics are globally asymptotically stable (cf. the phase portraits in Fig. 3), but the piecewise linear system (3.1) is not (see Fig. 4).

This example shows that stability is a real "hybrid problem," which requires novel stability analysis techniques beyond the large body of work on stability for continuous systems. In this survey, we will discuss two interesting questions with respect to stability of the switched system (2.1). First, we consider the case of stability under arbitrary switching and then stability for a given class of switching strategies. 


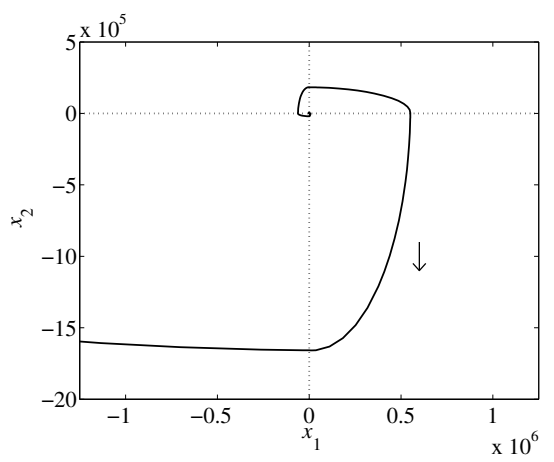

Figure 4. Unstable piecewise linear system (3.1).

(a) Stability under arbitrary switching

(i) General switched systems

We will say that the system (2.1) is globally uniformly asymptotically stable (GUAS), if there exists a $\mathcal{K} \mathcal{L}$ function $\beta \dagger$ such that for all switching signals $q$ and any initial state $x(0)$ the corresponding solutions $x$ of (2.1) satisfy the inequality

$$
\|x(t)\| \leq \beta(\|x(0)\|, t) \text { for all } t \geq 0
$$

where $\|x(t)\|:=\sqrt{\sum_{i=1}^{n} x_{i}^{2}(t)}$. Note that this implies that a switched system that is GUAS, has the properties $\lim _{t \rightarrow \infty} x(t)=0$ for all solutions of the system (attractivity) and $\|x(t)\| \leq \beta(\|x(0)\|, 0)$ for all $t \geq 0$ and thus for any arbitrary small neighborhood of 0 all trajectories can be guaranteed to stay in this neighborhood by taking $\|x(0)\|$ sufficiently small (Lyapunov stability). The terminology uniform is employed here to indicate the uniformity with respect to the switching signals: The bound (3.2) is independent of the particular choice of $q$.

Given a continuously differentiable $\left(C^{1}\right)$ function $V: \mathbb{R}^{n} \rightarrow \mathbb{R}$, we will say that it is a common Lyapunov function for the switched system (2.1), if $V$ is positive definite (i.e., $V(0)=0$ and $V(x)>0$ when $x \neq 0$ ) and

$$
L_{f_{i}} V(x):=\frac{\partial V}{\partial x} f_{i}(x)<0, \text { for all } x \neq 0 \text { and all } i=1, \ldots, N
$$

It is well established (Liberzon and Morse, 1999; Molchanov and Pyatnitskiy, 1989; Dayawansa and Martin, 1999) that if a common Lyapunov function exists for the switched system, then the switched system is GUAS as formalized in the next theorem.

Theorem 3.1. If the switched system (2.1) has a common Lyapunov function that is radially unbounded (i.e., $V(x) \rightarrow \infty$ when $\|x\| \rightarrow \infty$ ), then the switched system is GUAS.

As is the case for smooth systems also a converse Lyapunov result exists for switched systems under arbitrary switching. We assume that the functions $f_{i}$ in (2.1) are locally Lipschitz continuous.

$\dagger$ A function $\varphi: \mathbb{R}_{+} \rightarrow \mathbb{R}_{+}$belongs to class $\mathcal{K}$ if it is continuous, strictly increasing and $\varphi(0)=0$. A function $\beta: \mathbb{R}_{+} \times \mathbb{R}_{+} \rightarrow \mathbb{R}_{+}$belongs to class $\mathcal{K} \mathcal{L}$ if for each fixed $k \in \mathbb{R}_{+}, \beta(\cdot, k) \in \mathcal{K}$ and for each fixed $s \in \mathbb{R}_{+}, \beta(s, \cdot)$ is decreasing and $\lim _{k \rightarrow \infty} \beta(s, k)=0$. 
Theorem 3.2. (Mancilla-Aguilar and Garcia, 2000) If the switched system (2.1) is GUAS, then it has a radially unbounded common Lyapunov function.

Hence, GUAS (under arbitrary switching) of a switched systems is equivalent to the existence of a radially unbounded common Lyapunov function. However, in general it may be hard to construct such common Lyapunov functions. Nevertheless, for switched linear systems a particular class of common Lyapunov functions can be easily computed - provided there exists one.

\section{(ii) Switched linear systems}

Stability of switched linear systems of the form

$$
\dot{x}(t)=A_{q(t)} x(t)
$$

with $q(t) \in\{1, \ldots, N\}$ for all $t \in \mathbb{R}_{+}$and $A_{i} \in \mathbb{R}^{n \times n}, i \in\{1, \ldots, N\}$ can also be approached by the common Lyapunov approach and in imitation of linear systems one can aim to use a quadratic Lyapunov function of the form $V(x)=x^{\top} P x$ with $P$ a positive definite matrix $\dagger$. If the condition (3.3) is applied for a switched linear system using a quadratic Lyapunov function $V(x)=x^{\top} P x$, the following result is readily obtained.

Theorem 3.3. Suppose the set of linear matrix inequalities (LMIs)

$$
A_{i}^{\top} P+P A_{i} \prec 0 \text { for all } i=1, \ldots, N
$$

has a solution $P \succ 0$. Then the switched linear system (3.4) is GUAS.

It is important to note that the above LMI conditions are only sufficient for GUAS, but not necessary. Examples of switched linear systems exist that are GUAS, but do not allow a common quadratic Lyapunov function, see (Dayawansa and Martin, 1999). However, one can always show that if the switched linear system (3.4) is GUAS, then there is a common Lyapunov function of the form $V(x)=\max _{i=1,2, \ldots, k}\left(l_{i}^{\top} x\right)^{2}$ for certain constant vectors $l_{i}, i=1, \ldots, k$ (Meilakhs, 1978; Molchanov and Pyatnitskiy, 1989).

Under certain additional assumptions such as pairwise commutation of $A_{1}, \ldots$, $A_{N}$ (Narendra and Balakrishnan, 1994) or all matrices $A_{1}, \ldots, A_{N}$ being upper (or lower) triangularizable (Mori et al., 1997; Liberzon et al., 1999; Agrachev and Liberzon, 2001), one can actually show that a switched linear system is GUAS if and only if there exists a common quadratic Lyapunov function. Actually, under these additional assumptions, the switched linear systems is GUAS if and only if all matrices $A_{i}, i=1, \ldots, N$ are Hurwitz (all eigenvalues having strictly negative real parts).

\section{(b) Stability for classes of switching signals}

In various situations the switching strategy is already given. As such, the switching is not arbitrary, but belongs to a specific class. Of course, one can still apply

\footnotetext{
$\dagger$ We write $H \succ 0$ when $x^{\top} H x>0$ for all $x \neq 0$ (i.e., $H$ is positive definite) and $H \prec 0$ when $x^{\top} H x<0$ for all $x \neq 0$ (i.e., $H$ is negative definite.)
} 


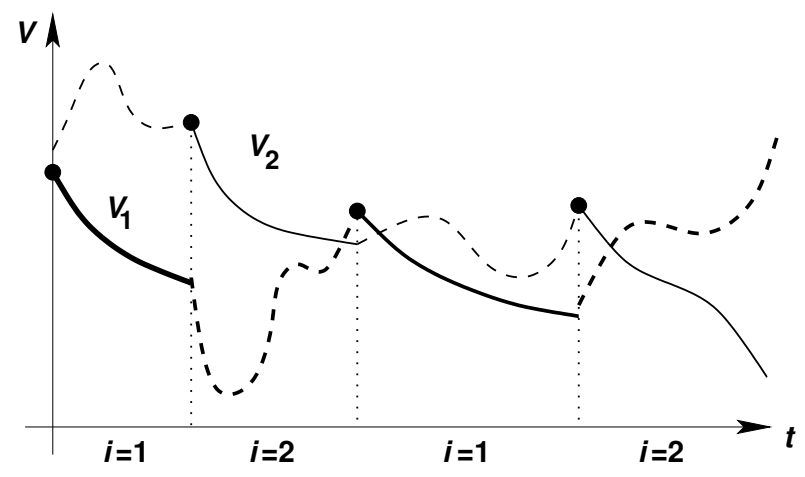

Figure 5. Multiple Lyapunov functions.

the results based on a common Lyapunov function as it shows stability for any switching signal, but one has to realize that such a test is conservative. In this section less conservative results will be presented for switched systems with either state-dependent or time-dependent switching. However, we will start with a general approach as proposed by (Branicky, 1998).

\section{(i) General approach based on multiple Lyapunov functions}

A general idea for the stability analysis of switched systems (2.1) is based on multiple Lyapunov functions (Branicky, 1998). The idea hinges on the fact that the underlying subsystems given by $\dot{x}=f_{i}(x), i=1, \ldots, N$, are globally asymptotically stable (GAS). Let $V_{i}, i=1, \ldots, N$, be their respective Lyapunov functions. Since we assume that no common Lyapunov function exists, this means that the switched system is not GUAS and (asymptotic) stability only holds for particular classes of switching signals $q$ (cf. Theorem 3.2). Let the switching times be given by $t_{k}, k=0,1,2, \ldots$ Note that the switching times are given by the times at which the switching signal $q$ is discontinuous. The question that arises now is for which switching signals / times global asymptotic stability (GAS) of the switched system can be proven.

In general the function $t \mapsto V_{q(t)}(x(t))$ will be discontinuous. Since $V_{i}$ is a Lyapunov function when submodel $i\left(\dot{x}=f_{i}(x)\right)$ is active, $V_{i}$ may increase when submodel $i$ is not active (See Figure 5). Without additional conditions this can still cause $V_{q(t)}(x(t))$ to go to infinity when $t \rightarrow \infty$ as in the piecewise linear (PWL) system (3.1). Hence, additional conditions are needed if we would like to guarantee GAS. In (Branicky, 1998), one proposed to put conditions on the values of the $i$ th Lyapunov function at the beginning of each time interval on which the $i$ th submodel becomes active as formulated in the next theorem.

Theorem 3.4. Consider the switched system (2.1) with all submodels $\dot{x}=f_{i}(x)$, $i=1, \ldots, N$, being $G A S$ and let $V_{i}, i=1, \ldots, N$, be corresponding Lyapunov functions. Suppose that there is a class $\mathcal{K}$ function $\rho$ with the property that for every pair of switching times $\left(t_{k}, t_{l}\right), k<l$ such that $q\left(t_{k}\right)=q\left(t_{l}\right)=i$ and $q\left(t_{m}\right) \neq i$ for any switching time $t_{m}$ with $t_{k}<t_{m}<t_{l}$, we have $V_{i}\left(x\left(t_{k}\right)\right)-V_{i}\left(x\left(t_{l}\right)\right) \leq$ $-\rho\left(\left\|x\left(t_{l}\right)\right\|\right)$, then the switched system is GAS for these sets of switching times. 
See (Branicky, 1998) for a proof. Note that in the formulation of the theorem we assume $q$ to be right continuous, i.e., $\lim _{t \downarrow t_{0}} q(t)=q\left(t_{0}\right)$ for all $t_{0}$.

In general this theorem is difficult to apply as we need to have information on the trajectories of the system at switching time instants. However, in the special case that the switching is induced in particular ways, life can become easier. For instance, when the switching is determined on the basis of the state variable only (e.g., as in (2.2)), one can try to impose continuity of the Lyapunov functions across the switching surfaces, as will be shown in the next section.

(ii) State-dependent switching: the case of piecewise linear systems

Consider piecewise linear systems given by

$$
\dot{x}=A_{i} x, \text { when } E_{i} x \geq 0, i \in\{1, \ldots, N\}
$$

where $x \in \mathbb{R}^{n}, A_{i} \in \mathbb{R}^{n \times n}$, and $E_{i} \in \mathbb{R}^{k_{i} \times n}, i=1, \ldots, N$. The inequality $E_{i} x \geq 0$ has to be interpreted componentwise and this defines a region $\mathcal{X}_{i}=\left\{x \in \mathbb{R}^{n} \mid\right.$ $\left.E_{i} x \geq 0\right\}$ in the state space $\mathbb{R}^{n}$ in which $\dot{x}=A_{i} x$ is active. We assume that the cells $\mathcal{X}_{i}, i \in\{1, \ldots, N\}$ form a partitioning of the state space in the sense that $\bigcup_{i=1}^{N} \mathcal{X}_{i}=\mathbb{R}^{n}$ and the cells $\mathcal{X}_{i}, i \in\{1, \ldots, N\}$ have pairwise disjoint interiors. Trajectories are assumed not to have sliding modes. Hence, we do only consider solutions to the system that satisfy for almost all times $t>0 \dot{x}(t)=A_{i} x(t)$ for some $i \in\{1, \ldots, N\}$ for which $x(t) \in \mathcal{X}_{i}$ holds. Moreover, we assume that there exist matrices $F_{i}, F_{j}$ such that $F_{i} x=F_{j} x$ for all $x \in \mathcal{X}_{i} \cap \mathcal{X}_{j}$. In (Johansson, 2003) the construction of $F_{i}, i=1, \ldots, N$, is discussed. These matrices play an essential role in guaranteeing continuity of the overall Lyapunov function.

There are several relaxations possible to the construction of a common quadratic Lyapunov function that would imply GUAS for arbitrary switching. First of all, one can require that the derivative $L_{f_{i}} V(x):=\frac{\partial V}{\partial x} f_{i}(x)$ of $V(x)=x^{\top} P x$ along $\dot{x}=f_{i}(x)$ is only negative in the region where the subsystem is active. Secondly, one can use multiple Lyapunov functions, say $V_{i}(x)=x^{\top} P_{i} x$ for each submodel as in Theorem 3.4. Finally, one can require that the Lyapunov function $V_{i}(x)=x^{\top} P_{i} x$ is only positive definite in its active region $\mathcal{X}_{i}$. These relaxations lead to the following theorem as given in (Johansson and Rantzer, 1998).

Theorem 3.5. If one can find symmetric matrices $T, W_{i}$ and $U_{i}$ with $U_{i}$ and $W_{i}$ having nonnegative entries and such that $P_{i}:=F_{i}^{\top} T F_{i}, i=1, \ldots, N$ satisfy the LMIs

$$
\begin{gathered}
A_{i}^{\top} P_{i}+P_{i} A_{i}+E_{i}^{\top} U_{i} E_{i} \prec 0, i=1, \ldots, N \\
P_{i}-E_{i}^{\top} W_{i} E_{i} \succ 0, i=1, \ldots, N
\end{gathered}
$$

then every continuous piecewise continuously differentiable trajectory (without sliding modes) of (3.6) tends to zero exponentially.

As indicated at the end of Section 3(i) the goal was to obtain a continuous Lyapunov function. Here, the Lyapunov function is chosen as $V(x)=x^{\top} P_{i} x=$ $x^{\top} F_{i}^{\top} T F_{i} x$ when $x \in \mathcal{X}_{i}$. Due to the property that was imposed on the matrices $F_{i}, i=1, \ldots, N$, we have that on the boundaries of the cells, e.g., $x \in \mathcal{X}_{i} \cap \mathcal{X}_{j}$, it holds that

$$
x^{\top} P_{i} x=x^{\top} F_{i}^{\top} T F_{i} x=x^{\top} F_{j}^{\top} T F_{j} x=x^{\top} P_{j} x
$$


thereby proving continuity of the Lyapunov function $V$. Since in each region strict decrease of $V$ is imposed by (3.7) and positive definiteness of $V$ is guaranteed by (3.8), global exponential stability and thus GAS can be proven for (3.6). The obtained conditions are only sufficient for GAS of (3.6), not necessary. Note that, in contrast with Theorem 3.1 and Theorem 3.4, the hypotheses of Theorem 3.5 do not require stability of the dynamics $\dot{x}=A_{i} x, i=1, \ldots, N$,

These basic ideas can be extended in various directions. For instance, piecewise affine systems were studied by Johansson (2003); Rantzer and Johansson (2000). The direct inclusion of the sliding modes in the LMI-based stability analysis was considered by Heemels and Weiland (2008). For discrete-time switched linear systems and piecewise affine systems the reader may consult (Ferrari-Trecate et al., 2002a; Daafouz et al., 2002; Johansson, 2003), where stability analysis methods based on multiple or piecewise quadratic (PWQ) Lyapunov functions are presented. Important subtleties in the (robust) stability analysis for discrete-time discontinuous and hybrid systems were recently exposed in (Lazar et al., 2009). Stability of piecewise polynomial systems and "polynomial hybrid systems" (including resets of the states) using a convex programming framework for the numerical computation of Lyapunov functions (based on sum-of-squares techniques) was given in (Papachristodoulou and Prajna, 2009). Other approaches that allow some memory in the switching (e.g., hybrid automata with affine dynamics in each modes and without resets) are studied in, e.g., (Johansson, 2003) and (Langerak et al., 2003).

\section{(iii) Time-dependent switching: Restricted dwell times}

Other classes of switching strategies can be characterized by dwell time restrictions on the switching signal $q$ in (2.1). The dwell time restrictions impose limitations on how long the switched system will be in a particular mode, see (Hespanha and Morse, 1999; Solo, 1994). For instance, one can adopt the minimal dwell time condition that imposes that two subsequent switching times are separated by at least $\tau_{d}>0$ time units. Of course, if the system is switching between GAS subsystems and $\tau_{d}$ is sufficiently large (for instance, to guarantee that the norm of the state vector at a switching time is strictly smaller than the norm of the state at the previous switching time as in line with the rationale of Theorem 3.4), GAS of (2.1) is obtained. In other words, if the switching between GAS subsystems is sufficiently slow, then the switched system is GAS as well. Here, we will actually consider a more general result based on average dwell time restrictions, which contains minimal dwell time conditions as a special case.

The concept of average dwell time was introduced by Hespanha and Morse (1999). To introduce this concept formally, let a switching signal $q: \mathbb{R}_{+} \rightarrow\{1, \ldots, N\}$ be given. The number of discontinuities of $q$ (switchings) on an interval $(t, T)$ is denoted by $N_{q}(t, T)$. We say that $q$ has the average dwell time $\tau_{a}$ if there exists a positive number $N_{0}$ such that

$$
N_{q}(t, T) \leq N_{0}+\frac{T-t}{\tau_{a}} \text { for all } T \geq t \geq 0
$$

Note that when $N_{0}=0$ this implies that $q$ has no discontinuities at all and hence, there is no switching. When $N_{0}=1$ we have that $q$ cannot switch twice on an interval of length smaller than $\tau_{a}$. Hence, in the latter case we recover the 
case of minimal dwell time $\tau_{d}=\tau_{a}$. Generally speaking, if we discard the first $N_{0}$ switchings, then the average time between consecutive switches is at least $\tau_{a}$.

Based on average dwell time restrictions, stability conditions of switched systems (2.1) are available:

Theorem 3.6. Consider the switched system (2.1). Suppose that there exist continuously differentiable $\left(\mathcal{C}^{1}\right)$ functions $V_{i}: \mathbb{R}^{n} \rightarrow \mathbb{R}_{+}, i=1, \ldots, N$ and constants $0<a<b, p \in \mathbb{N}, \mu \geq 1$, and $\lambda_{0}>0$ such that for all $i=1, \ldots, N$

- $a\|x\|^{p} \leq V_{i}(x) \leq b\|x\|^{p}$ for all $x \in \mathbb{R}^{n}$;

- $\frac{\partial V_{i}}{\partial x} f_{i}(x) \leq-\lambda_{0} V_{i}(x)$ for all $x \in \mathbb{R}^{n}$;

- $V_{i}(x) \leq \mu V_{j}(x)$ for all $i, j \in\{1, \ldots, N\}$ and all $x \in \mathbb{R}^{n}$.

Then the switched system (2.1) is globally asymptotically stable for every switching signal $q$ with average dwell time $\tau_{a}>\frac{\ln \mu}{\lambda_{0}}$ (and $N_{0}$ arbitrary).

See the book by (Liberzon, 2003) for the proof and more discussion on this interesting topic. The second condition in the above theorem states that $V_{i}$ is a Lyapunov function for the $i$ th subsystem $\dot{x}=f_{i}(x)$ and hence, all subsystems are necessarily GAS. Stability under dwell time conditions of switched systems with unstable subsystems was studied by Liu et al. (2009).

\section{(iv) Further stability results}

In case one is interested in systems with resets of the continuous state variable one might consider, next to the earlier mentioned work in (Papachristodoulou and Prajna, 2009), the results for hybrid inclusions (sometimes also called jump-flow systems) with flows $\dot{x} \in F(x)$ if $x \in \mathcal{C}$ and resets $x^{+} \in G(x)$ if $x \in \mathcal{D}$. The survey (Goebel et al., 2009) on hybrid inclusions is a good entry point to some (robust) stability theorems for such systems. Also the book by Haddad et al. (2006) on impulsive systems contains various interesting results on stability, dissipativity and beyond. For more information on stability analysis of hybrid systems one can also consult one of the surveys (Lin and Antsaklis, 2009; Shorten et al., 2007; Liberzon and Morse, 1999; DeCarlo et al., 2000).

\section{Control design}

In the previous section we have presented stability characterizations for various classes of hybrid systems. In this section we consider the stabilization problem that considers the issue of how to adapt or design a given hybrid system such that it becomes stable (given the design freedom). For instance, if there are free control inputs, one could pose the question of how to choose a controller for the hybrid system, which results in a stable closed-loop system.

\section{(a) LMI-based control}

Consider the switched linear system with added control inputs $u(t) \in \mathbb{R}^{m}, t \in \mathbb{R}$,

$$
\dot{x}(t)=A_{q(t)} x(t)+B_{q(t)} u(t), q(t) \in I=\{1, \ldots, N\}
$$


Suppose we would like to construct a feedback controller $u(t)=K_{q(t)} x(t)$ that renders the switched linear system GUAS (under arbitrary switching). This means that we have to find $K_{1}, \ldots, K_{N}$ such that

$$
\dot{x}(t)=\left(A_{q(t)}+B_{q(t)} K_{q(t)}\right) x(t)
$$

is GUAS. Note that this setup requires that we know the mode that is currently active as we schedule the feedback gains $K_{i}$ accordingly. Based on the previously mentioned characterizations for GUAS, a sufficient condition is to find a common quadratic Lyapunov function $V(x)=x^{\top} P x$ for some positive definite matrix $P$ such that

$$
\left(A_{i}+B_{i} K_{i}\right)^{\top} P+P\left(A_{i}+B_{i} K_{i}\right) \prec 0 \text { for all } i=1, \ldots, N \text { and } P \succ 0
$$

These equations can be transformed into LMIs by pre-multiplying and postmultiplying by $P^{-1}$ yielding

$$
P^{-1}\left(A_{i}+B_{i} K_{i}\right)^{\top}+\left(A_{i}+B_{i} K_{i}\right) P^{-1} \prec 0 \text { for all } i=1, \ldots, N \text { and } P^{-1} \succ 0
$$

which gives rise to the LMIs

$$
Z A_{i}^{\top}+A_{i} Z+Y_{i}^{\top} B_{i}^{\top}+B_{i} Y_{i} \prec 0 \text { for all } i=1, \ldots, N \text { and } Z \succ 0
$$

after the change of variables $Z:=P^{-1}$ and $Y_{i}:=K_{i} P^{-1}$. Once we have solved these LMIs $P$ can be recovered as $Z^{-1}$ and the stabilizing feedback gains are given by $K_{i}=Y_{i} Z^{-1}, i=1, \ldots, N$. In case the mode is not known, one can try to design a mode-independent stabilizing feedback $u(t)=K x(t)$ instead (by taking $K_{i}=K$, $i=1, \ldots, N$ and using $Y_{i}=Y, i=1, \ldots, N$ in the above procedure).

In the same spirit one might be interested in the stabilization of piecewise linear systems (3.6), with the control input $u$ added just as in (4.1), using the ideas of Theorem 3.5 together with the above transformations to obtain LMIs for the design of a PWL feedback stabilizing the system. Unfortunately, this approach results in design conditions formulated as bilinear matrix inequalities (BMIs), which are much harder to solve than LMIs. It is generally believed that these conditions cannot be transformed into LMIs.

\section{(b) Model predictive control}

One of the few general approaches to the design of controllers for hybrid systems in the discrete-time setting is model predictive control (MPC), which is based on solving optimal control problems. One of the nice features of MPC is that it can easily include control and state constraints. The only concerns, in particular in the hybrid context, are to guarantee that the resulting control law has favorable properties such as stability and robustness and to efficiently solve and implement the optimal control problems. Below we provide a brief introduction to the general setup of MPC and we refer the reader for more extensive information on MPC to, e.g., (Mayne et al., 2000; Maciejowski, 2002; Findeisen et al., 2003; Rawlings and Mayne, 2009). 
(i) $M P C$ setup

Although MPC can be based on many model types, let us focus for the sake of simplicity on discrete-time PWA systems of the form

$$
x_{k+1}=A_{q} x_{k}+B_{q} u_{k}+f_{q} \quad \text { when } x_{k} \in \mathcal{X}_{q}
$$

with the operation modes $q \in\{1, \ldots, N\} . A_{q} \in \mathbb{R}^{n \times n}, B_{q} \in \mathbb{R}^{n \times m}$, and $f_{q} \in \mathbb{R}^{n}$ are constant matrices and vectors for $q \in\{1, \ldots, N\}$ and $\mathcal{X}_{1}, \ldots, \mathcal{X}_{N}$ are convex polyhedra forming a partitioning of the set $\mathbb{X} \subseteq \mathbb{R}^{n}$ (i.e., $\mathbb{X}=\bigcup_{q} \mathcal{X}_{q}$ and the regions $\mathcal{X}_{q}, q \in\{1, \ldots, N\}$ do not overlap). The variable $x_{k} \in \mathbb{X} \subseteq \mathbb{R}^{n}$ is the state and $u_{k} \in \mathbb{U} \subseteq \mathbb{R}^{m}$ is the control action at the discrete-time instant $k \in \mathbb{N}$. Note that (4.4) is a discrete-time variant of (2.3). The sets $\mathbb{X}$ and $\mathbb{U}$ specify possible state and input constraints and it is assumed that they are compact polyhedral sets that contain the origin in their interiors. We assume for simplicity that $f_{q}=0$ for all $q \in\{1, \ldots, N\}$ for which the closure of $\mathcal{X}_{q}$ contains the origin 0 , which implies that the origin is an equilibrium state for (4.4) with $u_{k}=0$. The control goal is to stabilize this equilibrium by suitable selection of the control input $u_{k}$ at each time $k \in \mathbb{N}$ exploiting information on the state variable $x_{k}$.

MPC is based on using the model (4.4) and the current information of the state $x_{k}$ to predict the future behavior of the state $x$ over a prediction horizon $N \geq 1$, $N \in \mathbb{N}$, for choices of the future input sequence, and to select a future input sequence that minimizes some cost criterion $J$. In particular, let $\mathbf{x}_{k}\left(x_{k}, \mathbf{u}_{k}\right) \triangleq\left(x_{1 \mid k}, \ldots, x_{N \mid k}\right)$ denote the state sequence generated by system (4.4) from initial state $x_{0 \mid k} \triangleq x_{k}$ and by applying the input sequence $\mathbf{u}_{k}:=\left(u_{0 \mid k}, \ldots, u_{N-1 \mid k}\right) \in \mathbb{U}^{N}$ to $(4.4)$, where $\mathbb{U}^{N} \triangleq \mathbb{U} \times \ldots \times \mathbb{U}$. The class of admissible input sequences defined with respect to $\mathbb{X}_{T}$ and state $x_{k} \in \mathbb{X}$ is $\mathcal{U}_{N}\left(x_{k}\right) \triangleq\left\{\mathbf{u}_{k} \in \mathbb{U}^{N} \mid \mathbf{x}_{k}\left(x_{k}, \mathbf{u}_{k}\right) \in \mathbb{X}^{N}, x_{N \mid k} \in \mathbb{X}_{T}\right\}$, where we added a terminal constraint $x_{N \mid k} \in \mathbb{X}_{T}$ to guarantee favorable properties of the controlled system. $\mathbb{X}_{T} \subseteq \mathbb{X}$ is a desired target set that contains the origin in its interior, which has to be chosen in the design.

Problem 4.1. Let the target set $\mathbb{X}_{T} \subseteq \mathbb{X}$ and $N \in \mathbb{N}$ be given and let $F: \mathbb{R}^{n} \rightarrow \mathbb{R}_{+}$ with $F(0)=0$ and $L: \mathbb{R}^{n} \times \mathbb{R}^{m} \rightarrow \mathbb{R}_{+}$with $L(0,0)=0$ be mappings. At time $k \in \mathbb{N}$ let $x_{k} \in \mathbb{X}$ be given. Find $\mathbf{u}_{k}$ that minimizes the cost function $J\left(x_{k}, \mathbf{u}_{k}\right):=$ $F\left(x_{N \mid k}\right)+\sum_{i=0}^{N-1} L\left(x_{i \mid k}, u_{i \mid k}\right)$, with prediction model (4.4), over all input sequences $\mathbf{u}_{k} \in \mathcal{U}_{N}\left(x_{k}\right)$.

In the MPC literature, $F, L$ and $N$ are called the terminal cost, the stage cost and the prediction horizon, respectively. We call an initial state $x_{0} \in \mathbb{X}$ feasible if $\mathcal{U}_{N}\left(x_{0}\right) \neq \emptyset$. Let $\mathbb{X}_{f}(N) \subseteq \mathbb{X}$ denote the set of feasible initial states. We assume that there exists a unique optimal sequence of controls $\mathbf{u}_{k}^{*} \triangleq\left(u_{0 \mid k}^{*}, u_{1 \mid k}^{*}, \ldots, u_{N-1 \mid k}^{*}\right)$ for Problem 4.1 and any state $x_{k} \in \mathbb{X}_{f}(N)$. Then, the MPC control law is defined as

$$
u^{\mathrm{MPC}}\left(x_{k}\right) \triangleq u_{0 \mid k}^{*} ; \quad k \in \mathbb{N}
$$

Basically, the system (4.4) is controlled by the MPC law as in (4.5), which operates in a receding horizon fashion in the sense that at each time $k \in \mathbb{N}$ a new optimal control problem as in Problem 4.1 (depending on $x_{k}$ ) is solved providing an optimal control sequence. Only the first control move in this sequence is selected and actually implemented on the plant (4.4). At the next time $k+1 x_{k+1}$ is measured and the 
optimal control problem is solved again, but now for $x_{k+1}$. Interestingly, the above framework is quite flexible as it can be based on practically any model (as long as the solutions to the optimization problem can be computed fast enough) and can easily incorporate constraints on states and control inputs.

\section{(ii) Stability}

It is not always guaranteed that an MPC controller, although optimizing a performance criterion involving the state variable, indeed stabilizes the plant. For linear systems and smooth nonlinear systems several approaches are known that assure that particular MPC set-ups are globally $\dagger$ asymptotically stable (GAS), see, e.g., (Mayne et al., 2000). These are based on, for instance, putting an explicit condition such as $x_{N \mid k} \in \mathbb{X}_{T}$ and a weight $F$ in the cost criterion on the terminal state at the end of the prediction horizon. One such example is the use of an end-point equality constraint of the form $x_{N \mid k}=0$, i.e., $\mathbb{X}_{T}$ is taken to be $\{0\}$. Using such a condition in the MPC setup, a result on attractivity (all trajectories converging to the equilibrium state) was shown in (Bemporad and Morari, 1999) for the case where the plant (4.4) is replaced by a so-called mixed logical dynamical (MLD) model, which is given by linear equations and inequalities involving real and boolean variables. As proven in (Heemels et al., 2001) there exist strong relationships between MLD models and PWA models as in (4.4) (and other model classes). Although this attractivity result is of interest and one of the first within hybrid MPC, it is rather conservative in two ways. First of all, it relies on a terminal equality constraint, which is a severe condition as it requires the property that one can regulate any initial state of interest in finite time to 0 (this property is called (null) controllability), while weaker conditions (called stabilizability in control theory) should be sufficient. Secondly, the theorem only provides attractivity and not Lyapunov stability. As such, results that relax the terminal equality constraint to milder conditions such as, for instance, $x_{N \mid k} \in \mathbb{X}_{T}$, and guarantee Lyapunov stability are of interest. In (Lazar et al., 2006) conditions are provided for asymptotic stability (including Lyapunov stability) of discrete-time PWA systems in closed loop with MPC. However, due to the discontinuity of the corresponding closed-loop system and candidate Lyapunov function, such stability certificates may be fragile in the presence of arbitrarily small perturbations, as shown in (Lazar et al., 2009). Therefore, guarantees of robust stability, i.e., stability in the presence of perturbations, are of importance as well and these are studied in, e.g., (Lazar and Heemels, 2009).

\section{(iii) Implementation issues}

MPC is based on solving, in principle, an optimization problem as in Problem 4.1 at each time $k \in \mathbb{N}$. Due to the presence of the discrete modes this requires the solution of optimization problems involving both integers and real variables. In particular, in case of a discrete-time PWA system as in (4.4) and a cost criterion that consists of quadratic terms this results in mixed integer quadratic programming (MIQP) problems. If a linear cost criterion is used (involving for instance

\footnotetext{
$\dagger$ Globally indicates here that convergence to the equilibrium state 0 is guaranteed for each trajectory starting in any feasible initial state.
} 
1 - or $\infty$-norms) a mixed integer linear programming (MILP) problem has to be solved. Particularly convenient hybrid model formats for the formulation of hybrid MPC problems as MILP or MIQP are the earlier mentioned mixed logical dynamical (MLD) systems (Bemporad and Morari, 1999). More details on the conversion of Problem 4.1 for PWA or MLD models into MILP or MIQP problems can be found in (Bemporad and Morari, 1999). Using the relationships between MLD and PWA systems as in (Heemels et al., 2001) one can use the MLD modeling format also to describe PWA systems. In (Bemporad and Morari, 1999) branch-and-bound methods (Fletcher and Leyffer, 1998) are proposed to solve the resulting MPC optimization problems on-line, although other methods to solve them are also available. Unfortunately, MIQP and MILP problems are in general hard to solve (they are so-called "NP-hard") and problems with large dimensions can easily become computationally intractable for on-line implementation. Exploiting the possibly available structure in the optimization problem at hand to reduce the computational burden is then of interest. As an example, in (De Schutter and van den Boom, 2004) implementation issues are discussed for continuous PWA systems using the continuity of the right-hand side.

An important alternative approach to solving the optimization problems online is so-called explicit MPC in which the optimal feedback law as in (4.5) is solved explicitly as a function of $x_{k}$. Indeed, if the MPC-problem 4.1 gives rise to an MILP formulation, there exists an approach based on multi-parametric linear programming in which the MPC input as in (4.5) can be computed explicitly as a PWA function of $x_{k}$. This function can be computed explicitly off-line and stored in a look-up table. For the on-line control, one only then has to evaluate this PWA function, which has clear advantages over solving the MILP on-line at each discretetime step. However, the number of regions of the PWA function also increases rapidly as the size of the MPC problem increases, which also may render the explicit MPC computationally intractable. For more information on explicit MPC we refer the reader to (Bemporad et al., 2002a,c; Borrelli, 2003). It is of interest to mention that in case MPC is applied to a constrained linear system with quadratic costs the resulting explicit control law is also a PWA function (Bemporad et al., 2002b,c). In other words, the optimal control law for a constrained discrete-time linear systems with quadratic costs is a "hybrid" (namely PWA) state feedback. This indicates the importance of hybrid control also in the context of smooth systems.

\section{(c) Other control methods}

Given the space constraints of this survey paper, it is of course impossible to discuss all available hybrid control methodologies. Below we will provide a few additional pointers to some other approaches. In (Habets et al., 2006) an approach is given for controller synthesis of hybrid automata with affine dynamics in each mode, polyhedral invariants, and affine reset laws. This class of hybrid automata includes continuous-time PWA systems as a particular case. Based on computationally tractable conditions affine state feedback laws can be constructed that guarantee that the continuous state will leave the polyhedral invariant (region) corresponding to the affine dynamics of the present mode through a specific facet (edge) of the polyhedral region. The information of the (im)possibility is stored in a discreteevent abstraction (an automaton) that specifies which transitions can be guaranteed 
by proper choice of affine feedback. This discrete-event abstraction is then used to determine if one can steer the state of the system towards specific regions avoiding possible unsafe regions. This methodology is constructive in nature and was already used by other authors to solve (hybrid) control problems, see e.g., (Kloetzer and Belta, 2008). Related ideas can be found in (Tabuada, 2009). Other ideas for the design of stabilizing switching strategies for switched linear systems (although applicable only in a rather limited setting) are provided in (Wicks et al., 1998), see also, e.g., (Liberzon, 2003). Based on a convex combination of the matrices $A_{i}$ that is Hurwitz (if it exists), state-dependent and time-dependent switching laws can be designed, based on a quadratic Lyapunov function for the convex combination, that render the overall switched linear system globally asymptotically stable. Related to the above mentioned MPC approaches, which predominantly are studied in the discrete-time context, there are also optimal control approaches for continuoustime hybrid systems, see, e.g., (Cassandras et al., 2001; Branicky et al., 1998; Xu and Antsaklis, 2004; Rantzer and Johansson, 2000; De Schutter, 2000; Hedlund and Rantzer, 2002) and the references therein. Also hybrid extensions are available of the celebrated Pontryagin's maximum principle, giving necessary conditions for optimality in optimal control problems, see, e.g., (Sussmann, 1999; Garavello and Piccoli, 2005).

\section{Other control-related topics}

In this section we briefly discuss some other interesting control theoretic topics for hybrid systems.

Observer design and state estimation for hybrid systems is also a topic of interest as the full state variable is only rarely available. In this situation it might be convenient to obtain a good estimate of the (hybrid) state variable. Several papers are available on observer design for hybrid systems, especially in the context of switched and piecewise linear systems. The problem turns out to be complicated, especially when the mode of the system is not known or cannot be directly reconstructed on the basis of the available measurements. The situation becomes even more complicated when resets of the continuous state variable are present in the hybrid system at hand. Good starting points for investigating hybrid observers are, for instance, (Alessandri and Coletta, 2001; Bara et al., 2001; Juloski et al., 2007; Petterson, 2005; Balluchi et al., 2002; Ferrari-Trecate et al., 2002b; van de Wouw and Pavlov, 2008) and the references therein.

As observed above, the stability analysis and controller design are all modelbased, which means that systematic identification methods to obtain appropriate hybrid models on the basis of measured data are indispensable. Some approaches for the identification of hybrid systems are described in (Juloski et al., 2005b; FerrariTrecate et al., 2003; Roll et al., 2004; Vidal et al., 2003; Bemporad et al., 2005), while a comparison between various identification procedures is given in (Juloski et al., 2005a). The comparison paper also presents important problems that hybrid identification methods have to face.

In section 3 the fundamental properties of well-posedness (existence and uniqueness of trajectories given an initial condition and external signals) was briefly mentioned. More information on this challenging subject can be found in (Heemels et al., 2003; Cortes, 2008). Other fundamental properties of hybrid systems are controlla-

Preprint for Royal Society 
bility, stabilizability, observability and detectability, which have played a central role throughout the history of modern control theory. The complexity of characterizing controllability and stabilizability for hybrid systems has been studied in (Blondel and Tsitsiklis, 1999). In these papers it was shown that even for very elementary classes of hybrid systems there is no algorithm to decide the controllability status of a given system. Hence, there is no hope to find complete conditions that characterize these properties for general hybrid systems. The best one can aim for are characterizations for some specific classes of hybrid systems. In this context controllability problems for piecewise linear systems and various related model classes have been studied by (Camlibel et al., 2008; Lee and Arapostathis, 1987; Bemporad et al., 2000; Blondel and Tsitsiklis, 1999; Habets and van Schuppen, 2001) to mention a few. A similar story holds for observability and detectability (Bemporad et al., 2000; Camlibel et al., 2006; Babaali and Egerstedt, 2004; Collins and van Schuppen, 2004).

\section{Concluding remarks}

The aim of this survey was to provide an overview of representative results in hybrid systems theory as obtained by the control community. Clearly, a survey written by computer scientists might have emphasized different aspects of hybrid systems and we refer the interested reader to the references in this survey to get a broader and more in-depth perspective on hybrid systems. In this way, we hope that this survey can serve as a good starting point to learn more about the different modeling, analysis and control design techniques that are available for hybrid systems.

It is without any dispute that the field of hybrid systems will grow in the near future due to the rich application fields ranging from man-made (technological) systems to naturally occurring physical systems exhibiting hybrid behavior, as is evidenced by the many interesting papers in this special issue. In particular, the current interest in networked control systems will spur the application and development of hybrid systems theory even further in the years to come as communication networks introduce additional discrete phenomena (scheduling protocols, message loss, etc.) in the control of physical systems.

\section{References}

A.A. Agrachev and D. Liberzon. Lie-algebraic stability criteria for switched systems. SIAM Journal on Control and Optimization, 40(1):253-269, 2001.

A. Alessandri and P. Coletta. Design of Luenberger observers for a class of hybrid linear systems. In Proc. of Hybrid Systems: Computation and Control, volume 2034 of Lecture Notes in Computer Science, pages 7-18, Rome, 2001. Springer.

R. Alur and D. Dill. A theory of timed automata. Theoretical Computer Science, 126:183-235, 1994.

R. Alur and T.A. Henzinger. Modularity for timed and hybrid systems. In Proceedings of the 8th International Conference on Concurrency Theory, volume 1243 of Lecture Notes in Computer Science, pages 74-88. Springer Verlag, 1997. 
R. Alur, C. Courcoubetis, N. Halbawachs, T.A. Henzinger, P.H. Ho, X. Nicollin, A. Olivero, J. Sifakis, and S. Yovine. The algorithmic analysis of hybrid systems. Theoretical Computer Science, 138:3-34, 1995.

P.J. Antsaklis and A. Nerode. Special issue on hybrid control systems. IEEE Trans. Autom. Control, 43(4), 1998.

M. Babaali and M. Egerstedt. Observability for switched linear systems. In Hybrid Systems: Computation and Control, volume 2623 of Lecture Notes in Computer Science. Springer, 2004.

A. Balluchi, L. Benvenuti, M. D. Di Benedetto, and A. L. Sangiovanni-Vincentelli. Design of observers for hybrid systems. In C.J. Tomlin and J.R. Greenstreet, editors, Hybrid Systems: Computation and Control, volume 2289 of Lecture Notes in Computer Science, pages 76-89. Springer-Verlag, Stanford, CA, 2002.

G.I. Bara, J. Daafouz, F. Kratz, and J. Ragot. Parameter dependent state observer design for affine LPV systems. International Journal of Control, 74(16):1601$1611,2001$.

A. Bemporad and M. Morari. Control of systems integrating logic, dynamics, and constraints. Automatica, 35:407-427, 1999.

A. Bemporad, G. Ferrari-Trecate, and M. Morari. Observability and controllability of piecewise affine and hybrid systems. IEEE Trans. Autom. Control, 45(10): 1864-1876, 2000.

A. Bemporad, F. Borrelli, and M. Morari. Model predictive control based on linear programming - The explicit solution. IEEE Trans. Autom. Control, 47(12): 1974-1985, 2002a.

A. Bemporad, W.P.M.H. Heemels, and B. De Schutter. On hybrid systems and closed-loop MPC systems. IEEE Trans. Autom. Control, 47(5):863-869, 2002b.

A. Bemporad, M. Morari, V. Dua, and E.N. Pistikopoulos. The explicit linear quadratic regulator for constrained systems. Automatica, 38(1):3-20, January 2002c.

A. Bemporad, A. Garulli, S. Paoletti, and A. Vicino. A bounded-error approach to piecewise affine system identification. IEEE Trans. Autom. Control, 50(10): $1567-1580,2005$.

V.D. Blondel and J.N. Tsitsiklis. Complexity of stability and controllability of elementary hybrid systems. Automatica, 35(3):479-490, 1999.

F. Borrelli. Constrained Optimal Control of Linear and Hybrid Systems, volume 290 of Lecture Notes in Control and Information Sciences. Springer, Berlin, 2003.

M.S. Branicky. Multiple Lyapunov functions and other analysis tools for switched and hybrid systems. IEEE Trans. Autom. Control, 43(4):475-482, 1998.

M.S. Branicky, V.S. Borkar, and S.K. Mitter. A unified framework for hybrid control: model and optimal control theory. IEEE Trans. Autom. Control, 43(1): 31-45, 1998.

Preprint for Royal Society 
B. Brogliato. Nonsmooth Impact Mechanics. Models, Dynamics and Control, volume 220 of Lecture Notes in Control and Information Sciences. Springer, London, 1996.

M.K. Camlibel, J.S. Pang, and J. Shen. Conewise linear systems: non-zenoness and observability. SIAM J. Control Optimizaton, 45:1769-1800, 2006.

M.K. Camlibel, W.P.M.H. Heemels, and J.M. Schumacher. Algebraic necessary and sufficient conditions for the controllability of conewise linear systems. IEEE Trans. Autom. Control, 53(3):762-774, 2008.

C. Cassandras and J. Lygeros. Stochastic Hybrid Systems. CRC Taylor \& Francis, Control Engineering Series, 2007.

C.G. Cassandras and S. Lafortune. Introduction to Discrete Event Systems. Kluwer Academic Publishers, Boston, 1999.

C.G. Cassandras, D.L. Pepyne, and Y. Wardi. Optimal control of a class of hybrid systems. IEEE Trans. Autom. Control, 46(3):398-415, 2001.

P. Collins and J.H. van Schuppen. Observability of piecewise-affine hybrid systems. In Hybrid Systems: Computation and Control, volume 2993 of Lecture Notes in Computer Science, pages 265-279. Springer Verlag, Berlin, 2004.

J. Cortes. Discontinuous dynamical systems. IEEE Control Systems Magazine, 28: 36-73, 2008.

J. Daafouz, P. Riedinger, and C. Iung. Stability analysis and control synthesis for switched systems: A switched Lyapunov function approach. IEEE Trans. Autom. Control, 47:1883-1887, 2002.

W.P. Dayawansa and C.F. Martin. A converse Lyapunov theorem for a class of dynamical systems which undergo switching. IEEE Trans. Autom. Control, 44 (4):751-760, 1999.

B. De Schutter. Optimal control of a class of linear hybrid systems with saturation. SIAM J. Control Optim., 39(3):835-851, 2000.

B. De Schutter and T.J.J. van den Boom. MPC for continuous piecewise-affine systems. Systems \& Control Letters, 52(3-4):179-192, 2004.

R. DeCarlo, M. Branicky, S. Pettersson, and B. Lennartson. Perspectives and results on the stability and stabilizability of hybrid systems. Proc. of the IEEE, pages 1069-1082, 2000.

G. Ferrari-Trecate, F. A. Cuzzola, D. Mignone, and M. Morari. Analysis of discretetime piecewise affine and hybrid systems. Automatica, 38(12):2139-2146, 2002a.

G. Ferrari-Trecate, D. Mignone, and M. Morari. Moving horizon estimation for hybrid systems. IEEE Trans. Autom. Control, 47:1663-1676, $2002 \mathrm{~b}$.

G. Ferrari-Trecate, M. Muselli, D. Liberati, and M. Morari. A clustering technique for the identification of piecewise affine systems. Automatica, 39(2):205-217, 2003. 
A.F. Filippov. Differential Equations with Discontinuous Righthand Sides. Mathematics and its Applications. Kluwer, The Netherlands, 1988.

R. Findeisen, L. Imsland, F. Allgower, and B.A. Foss. State and output feedback nonlinear model predictive control: An overview. European Journal of Control, 9:190-206, 2003.

R. Fletcher and S. Leyffer. Numerical experience with lower bounds for MIQP branch-and-bound. SIAM Journal on Optimization, 8(2):604-616, 1998.

M. Garavello and B. Piccoli. Hybrid necessary principle. SIAM J. Control and Optimization, 43:1867-1887, 2005.

R. Goebel and A.R. Teel. Solution to hybrid inclusions via set and graphical convergence with stability theory applications. Automatica, 42:573-587, 2006.

R. Goebel, J. Hespanha, A. Teel, C. Cai, and R. Sanfelice. Hybrid systems: generalized solutions and robust stability. In Proc. IFAC Symposium on Nonlinear Control Systems (NOLCOS), pages 1-12, Stuttgart, Germany, 2004.

R. Goebel, R. Sanfelice, and A.R. Teel. Hybrid dynamical systems. IEEE Control Systems Magazine, 29(2):28-93, 2009.

L.C.G.J.M. Habets and J.H. van Schuppen. A controllability result for piecewiselinear hybrid systems. In Proc. 2001 European Control Conference, Porto, Portugal, 2001.

L.C.G.J.M. Habets, P.J. Collins, and J.H. van Schuppen. Reachability and control synthesis for piecewise-affine hybrid systems on simplices. IEEE Trans. Autom. Control, 51:938-948, 2006.

W.M. Haddad, V. Chellaboina, and S.G. Nersesov. Impulsive and Hybrid Dynamical Systems: Stability, Dissipativity, and Control. Princeton University Press, 2006.

S. Hedlund and A. Rantzer. Convex dynamic programming for hybrid systems. IEEE Trans. Autom. Control, 47(9):1536-1540, 2002.

W.P.M.H. Heemels and S. Weiland. Input-to-state stability and interconnections of discontinuous dynamical systems. Automatica, 44:3079-3086, 2008.

W.P.M.H. Heemels, B. De Schutter, and A. Bemporad. Equivalence of hybrid dynamical models. Automatica, 37(7):1085-1091, 2001.

W.P.M.H. Heemels, M.K. Camlibel, A.J. van der Schaft, and J.M. Schumacher. On the existence and uniqueness of solution trajectories to hybrid dynamical systems. In Nonlinear and Hybrid Control in Automotive Applications. Editors: R. Johansson and A. Rantzer, pages 391-422. Springer, 2003.

T.A. Henzinger and S. Sastry, editors. Proc. First International Workshop on Hybrid Systems: Computation and Control, volume 1386 of Lecture Notes In Computer Science. Springer, 1998.

Preprint for Royal Society 
J.P. Hespanha and A.S. Morse. Stability of switched systems with average dwelltime. In Proc. 38th IEEE Conference on Decision and Control, pages 2655-2660, 1999 .

M. Johansson. Piecewise Linear Control Systems, volume 284 of Lecture Notes in Control and Information Sciences. Springer, Berlin, Germany, 2003.

M. Johansson and A. Rantzer. Computation of piecewise quadratic Lyapunov functions for hybrid systems. IEEE Trans. Autom. Control, 43(4):555-559, 1998.

A. Lj. Juloski, W.P.M.H. Heemels, G. Ferrari-Trecate, R. Vidal, S. Paoletti, and J.H.G. Niessen. Comparison of four procedures for the identification of hybrid systems. In Hybrid Systems: Computation and Control, volume 3414 of Lecture Notes in Computer Science, pages 354-369. Springer Verlag, 2005a.

A. Lj. Juloski, S. Weiland, and W.P.M.H. Heemels. A Bayesian approach to identification of hybrid systems. IEEE Trans. Autom. Control, 50(10):1520-1533, 2005b.

A.Lj. Juloski, W.P.M.H. Heemels, and S. Weiland. Observer design for a class of piecewise linear systems. Intern. J. Robust and Nonlinear Control, 17(15): 1387-1404, 2007.

H.K. Khalil. Nonlinear Systems, 2nd ed. Prentice Hall, Upper Saddle River, 1996.

M. Kloetzer and C. Belta. A fully automated framework for control of linear systems from temporal logic specifications. IEEE Trans. Autom. Control, 53(1):287-297, 2008 .

R. Langerak, J.W. Polderman, and T. Krilavičius. Stability analysis for hybrid automata using conservative gains. In Proceedings of IFAC Conference on Analysis and Design of Hybrid Systems, pages 337-342, Saint Malo, France, 2003.

M. Lazar and W.P.M.H. Heemels. Predictive control of hybrid systems: Inputto-state stability results for sub-optimal solutions. Automatica, 45(1):180-185, 2009 .

M. Lazar, W.P.M.H. Heemels, S. Weiland, and A. Bemporad. Stabilizing model predictive control of hybrid systems. IEEE Trans. Autom. Control, 51(11):1813$1818,2006$.

M. Lazar, W. P. M. H. Heemels, and A. R. Teel. Lyapunov functions, stability and input-to-state stability subtleties for discrete-time discontinuous systems. IEEE Trans. Autom. Control, 54:2421-2425, 2009.

K.K. Lee and A. Arapostathis. On the controllability of piecewise-linear hypersurface systems. Systems \& Control Letters, 9:89-96, 1987.

D. Liberzon. Switching in Systems and Control. Systems \& Control: Foundations and Applications. Birkhäuser, Boston, Massachusetts, 2003.

D. Liberzon and A.S. Morse. Basic problems in stability and design of switched systems. IEEE Control Systems Magazine, 19(5):59-70, 1999. 
D. Liberzon, J.P. Hespanha, and A.S. Morse. Stability of switched systems: a Liealgebraic condition. Systems \& Control Letters, 37:117-122, 1999.

H. Lin and P. J. Antsaklis. Stability and stabilizability of switched linear systems: A survey of recent results. IEEE Trans. Autom. Control, 54(2):308-322, 2009.

J. Liu, X. Liu, and W.-C. Xie. Uniform stability of switched nonlinear systems. Nonlinear Analysis: Hybrid Systems, 3(4):441-454, 2009.

J. Lunze and F. Lamnabhi-Lagarrigue. Handbook of Hybrid Systems Control: Theory, Tools, Applications. Cambridge University Press, 2009.

J. Lygeros, D.N. Godbole, and S. Sastry. Verified hybrid controllers for automated vehicles. IEEE Trans. Autom. Control, 43:522-539, 1998.

N. Lynch, R. Segala, F. Vaandrager, and H.B. Weinberg. Hybrid I/O automata. In Hybrid Systems III, volume 1066 of Lecture Notes in Computer Science, pages 496-510. Springer Verlag, 1996.

J.M. Maciejowski. Predictive Control with Constraints. Prentice Hall, Harlow, England, 2002.

J.L. Mancilla-Aguilar and R.A. Garcia. A converse Lyapunov theorem for nonlinear switched systems. Systems $\&$ Control Letters, 41:67-71, 2000.

D. Q. Mayne, J.B. Rawlings, C.V. Rao, and P.O.M. Scokaert. Constrained model predictive control: Stability and optimality. Automatica, 36(6):789-814, 2000.

A.M. Meilakhs. Design of stable control systems subject to parametric perturbation. Automat. Remote Control, 39:1409-1418, 1978.

A.P. Molchanov and Y.S. Pyatnitskiy. Criteria of absolute stability of differential and difference inclusions encountered in control theory. Systems \& Control Letters, 13:59-64, 1989.

Y. Mori, T. Mori, and Y. Kuroe. A solution to the common Lyapunov function problem for continuous-time systems. In Proc. 36th IEEE Conference on Decision and Control, pages 3530-3531, San Diego, Ca., 1997.

A.S. Morse, S.S. Pantelides, S. Sastry, and J.M. Schumacher. A special issue on hybrid systems. Automatica, 35(3), 1999.

K.S. Narendra and J. Balakrishnan. A common Lyapunov function for stable LTI systems with commuting A-matrices. IEEE Trans. Autom. Control, 39(12):2469$2471,1994$.

A. Papachristodoulou and S. Prajna. Robust stability analysis of nonlinear hybrid systems. IEEE Trans. Autom. Control, 54(5):1035-1041, 2009.

S. Petterson. Switched state jump observers for switched systems. In Proc. IFAC World Congress, Prague, Czech Republic, 2005.

A. Pnueli and J. Sifakis. Special issue on hybrid systems. Theoretical Computer Science, 138, 1995.

Preprint for Royal Society 
A. Rantzer and M. Johansson. Piecewise linear quadratic optimal control. IEEE Trans. Autom. Control, 45(4):629-637, 2000.

J.B. Rawlings and D.Q. Mayne. Model Predictive Control: Theory and Design. Nob Hill Publishing, Madison, Wisconsin, 2009.

J. Roll, A. Bemporad, and L. Ljung. Identification of piecewise affine systems via mixed-integer programming. Automatica, 40(1):37-50, 2004.

R. Shorten, F. Wirth, O. Mason, K. Wulff, and C. King. Stability criteria for switched and hybrid systems. SIAM Review, 49(4):545-592, 2007.

V. Solo. On the stability of slowly time-varying linear systems. Mathematics of Control, Signals and Systems, 7:331-350, 1994.

E.D. Sontag. Nonlinear regulation: the piecewise linear approach. IEEE Trans. Autom. Control, 26(2):346-357, 1981.

E.D. Sontag. Interconnected automata and linear systems: a theoretical framework in discrete-time. In Hybrid Systems III, volume 1066 of Lecture Notes in Computer Science, pages 436-448. Springer, 1996.

H.J. Sussmann. A maximum principle for hybrid optimal control problems. In Proc. 38th IEEE Conference on Decision and Control, pages 425-430, 1999.

P. Tabuada. Verification and Control of Hybrid Systems: A Symbolic Approach. Springer, 2009.

N. van de Wouw and A. Pavlov. Tracking and synchronisation for a class of PWA systems. Automatica, 44(11):2909-2915, 2008.

A. J. van der Schaft and J. M. Schumacher. The complementary-slackness class of hybrid systems. Mathematics of Control, Signals and Systems, 9:266-301, 1996.

A.J. van der Schaft and J.M. Schumacher. An Introduction to Hybrid Dynamical Systems, volume 251 of LNCIS. Springer-Verlag, London, 2000.

A.J. van der Schaft and J.M. Schumacher. Complementarity modeling of hybrid systems. IEEE Trans. Autom. Control, 43:483- 490, 1998.

R. Vidal, S. Soatto, and S. Sastry. An algebraic geometric approach for identification of linear hybrid systems. In Proc. 42nd IEEE Conference on Decision and Control, 2003.

M. Wicks, P. Peleties, and R. DeCarlo. Switched controller synthesis for the quadratic stabilization of a pair of unstable linear systems. Eur. J. Contr., 4: 140-147, 1998.

H.S. Witsenhausen. A class of hybrid-state continuous-time dynamic systems. IEEE Trans. Autom. Control, 11(2):161-167, 1966.

$\mathrm{X}$. Xu and P.J. Antsaklis. Optimal control of switched systems based on parameterization of the switching instants. IEEE Trans. Autom. Control, 49(1):2-16, 2004 . 